\title{
PREVENCIÓN DE EMOCIONES NEGATIVAS EN LA ADOLESCENCIA: VALORACIÓN DE TÉCNICAS COGNITIVO-CONDUCTUALES
}

\author{
MARGARITA OLMEDO ${ }^{1}$, VICTORIA DEL BARRIO² y MIGUEL Á. SANTED ${ }^{2}$ \\ ${ }^{1}$ Universidad de Jaén \\ ${ }^{2}$ Universidad Nacional de Educación a Distancia (UNED)
}

(Recibido el 6 de abril de 1998)

\begin{abstract}
En este trabajo se presentan los datos acerca de la eficacia que posee la enseñanza de diferentes estrategias (entrenamiento en autoestima, relajación, habilidades sociales y solución de problemas) para disminuir los niveles de depresión y ansiedad en población no clínica adolescente. Los estudios epidemiológicos realizados en las sociedades desarrolladas muestran un incremento de los problemas emocionales en esta etapa de la vida y por tanto, la oportunidad de su prevención y solución. Para evaluar la efectividad de la intervención contamos con una muestra formada por 225 adolescentes con una edad media de 13,22 (DT=0,54), distribuidos en dos grupos, uno de intervención y otro de comparación. Ambos equiparables en cuanto a edad, sexo y características socioculturales. Los análisis estadísticos realizados, con la finalidad de determinar el peso predictivo de cada uno de los módulos que componen el programa respecto a la disminución de ansiedad y depresión, reflejan la superioridad del entrenamiento en relajación y en habilidades sociales para la disminución de los niveles de ansiedad. En el caso de la depresión, el único módulo de intervención que no alcanzó valores predictivos significativos, en relación con la disminución de dicha variable, fue el entrenamiento en habilidades sociales.
\end{abstract}

Palabras clave: Técnicas cognitivo-conductuales, evaluación de programas, adolescencia, depresión y ansiedad.

\section{Prevention of negative emotions in Adolescence: Evaluation of cognitive-behavioral techniques}

This study presents data about the efficacy of teaching different strategies (training in self-esteem relaxation, social skills training and problem solving) in order to diminish depression and anxiety levels in an adolescent general population sample. Epidemiological studies performed on developed societies show an increase of emotional problems at this stage of life. The goal of our work is the assessment and solution of these problems. To assess the effectivity of the intervention, we applied a prevention program on a sample formed by 225 adolescents with an average age of 13,22 belonging to two different groups: intervention and comparation. Both of groups have similar age, sex and Social Economical State (SES). A statistical analyses was carried out in order to determine the predictive weight of each of the program's modules in decreasing anxiety and depression levels. Our results show that anxiety levels are reduced through relaxation training and social skills improvement. On the other hand, social skills improvement was the only type of intervention that had no effect in decreasing depression levels.

Key words: Cognitive-behavioral techniques, program's evaluation, adolescence, depression, anxiety. 


\section{INTRODUCCIÓN}

Existe una certeza creciente de que las prevalencias de desórdenes emocionales están sufriendo un incremento constante en la población adolescente. Si bien en un tiempo se creía que la depresión y la ansiedad eran perturbaciones típicas de la mediana edad, los estudios realizados en edades inferiores han demostrado que los problemas de depresión, al igual que los de ansiedad, pueden ocurrir, y de hecho ocurren, mucho antes de alcanzar la etapa adulta (Brady y Kendall, 1992; Fergusson, Horwood y Lynskey, 1995; Lefkovitz y Tesiny, 1981; Polaino, 1987; Reinherz, Frost y Pakiz, 1991; Rutter, 1986). Incluso hoy se tiene la convicción de que son los sujetos adolescentes y adultos muy jóvenes los que están alcanzando las incidencias más altas en este tipo de trastornos (Kandel y Davies, 1986). Así mismo, numerosas investigaciones han puesto de manifiesto reiteradamente, que se produce un aumento de las alteraciones emocionales con el paso de la niñez a la adolescencia (Del Barrio, 1990; Del Barrio, Frías y Mestre, 1994; Mestre, 1992; Oster y Caro, 1990; Rutter, 1989).

Si a estas observaciones añadimos el hecho, igualmente constatado, de que los problemas emocionales, en esta etapa de la vida, predicen futuros problemas de adaptación, así como un incremento en la probabilidad de sufrir este tipo de trastornos en la edad adulta (Gröer, Thomas y Shoffer, 1992), se hace evidente la necesidad de profundizar en el conocimiento de la depresión y la ansiedad entre los adolescentes y de los factores que puedan actuar sobre estos problemas, con el fin de poner en marcha programas de intervención orientados a una prevención primaria. Desde la presente investigación se pretende aportar alguna contribución en este sentido.

A partir del conocimiento obtenido en estudios anteriores (Del Barrio, 1997), donde se ha investigado la relación entre las estrategias de afrontamiento utilizadas por los adolescentes y los niveles de depresión y ansiedad autoinformados, podemos afirmar que el aprendizaje de recursos o estrategias de afrontamiento adecuadas adquiere especial relevancia de cara a facilitar la adaptación y proteger del impacto emocional que los diferentes estresores puedan provocar en esta etapa de la vida.

Así pues, bajo esta premisa y con la intención de configurar un programa de intervención dedicado a la enseñanza de técnicas de afrontamiento que resultaran efectivas a estos niveles de edad para prevenir los problemas emocionales, tuvimos en cuenta el alto consenso entre los expertos en este tema al considerar las estrategias cognitivo-conductuales como las más apropiadas para realizar un programa de intervención que cumpliera tales objetivos.

Fundamentándonos en las propuestas realizadas por diferentes autores que anteriormente se han ocupado del tratamiento de la ansiedad y depresión infanto-juvenil, realizamos la configuración de nuestro programa. La diferencia más relevante es que, en esta ocasión, la intervención se ubicó en un nivel preventivo con población no clínica, tratando de demostrar su efectividad en este tipo de circunstancias. El trabajo se centró en la enseñanza de aquellas estrategias que han demostrado ser más efectivas en anteriores investigaciones (Cautela y Groden, 1985; Mackay, Davis y Fanning, 1981; Michelson et al., 1984; Reynolds y Coats, 1986; Shaffi y Shaffi, 1992; Spivack y Shure, 1974; Vostanis y Harrington, 1994), sin utilizar otras más concretas o personalizadas, ya que, lo que se pretendía era valorar la utilidad de un programa de aplicación general, y una vez comprobada su efectividad, pensar en la posibilidad de hacerlo extensible a otras poblaciones con las mismas características. 


\section{Programa de intervención}

La intervención a evaluar estuvo constituida por cuatro módulos dedicados a conseguir en el adolescente: 1) un aumento de su autoestima, 2) un buen aprendizaje en técnicas de relajación, 3) la elevación en su nivel de habilidades sociales y 4) incrementar la capacidad de solución de problemas personales.

Dichos módulos, implantados por este orden, se encontraban distribuidos en 18 sesiones, de una hora de duración cada una y con frecuencia semanal. La justificación teórica que subyace a la implantación de los mismos, así como una descripción pormenorizada de la forma en que han sido llevados a cabo, figuran en el «Programa de afrontamiento de problemas emocionales en adolescentes», de Olmedo, Del Barrio y Santed (en prensa).

El lugar elegido para la realización de este programa es el centro escolar, ya que este es el marco de referencia más representativo donde el adolescente configura su propia imagen, tiene que mostrar sus capacidades, competir y afiliarse con sus compañeros, estando su ejecución continuamente evaluada tanto a nivel social como académico.

En este punto, de acuerdo con Pelechano (1996), habría que reconocer que, en el ámbito escolar, no se presta la suficiente atención a los hábitos de afrontamiento para los problemas personales y sociales, que son tan importantes en la vida del adolescente. Discusiones relacionadas con la autoestima, el autocontrol, la forma de relacionarse con los demás y de enfrentarse a los problemas, pueden formar parte de los temas a tratar en clase, al menos en las horas dedicadas a tutorías (Butler, Miezitis, Frieman y Cole, 1980). El programa que aquí se presenta pretende ser una aportación a dicha tarea educativa.

En la actualidad, hay numerosos programas en marcha en el ámbito educativo, sin embargo muy pocos son sometidos a evaluación. Por ello, una de las preocupaciones que nos planteamos fue la elaboración de un diseño metodológicamente adecuado, que permitiese dotar al presente trabajo del carácter científico propio de todo proyecto que pretenda alcanzar una utilidad social, y a través del cual pudiésemos determinar la eficacia de la intervención, objetivo que se ha considerado fundamental en el presente trabajo.

Igualmente, consideramos relevante en nuestro estudio conocer el peso diferencial de las diferentes técnicas aplicadas en la eficacia para disminuir los niveles de depresión y ansiedad en los adolescentes, dado que consideramos la posibilidad de que algunos módulos presentasen un mayor poder explicativo respecto al cambio producido por el programa o afectasen exclusivamente a una de las variables evaluadas (depresión o ansiedad rasgo). En este sentido podemos considerar nuestro trabajo exploratorio.

\section{MÉTODO}

Sujetos

Para cumplir los mencionados objetivos trabajamos con una muestra formada por 225 adolescentes (103 del género masculino y 122 del femenino), cuyo rango de edad se sitúa entre los 12 y los 16 años al comienzo del estudio $(M=$ $13,22$ y $D T=0,54)$, todos ellos pertenecientes al nivel escolar de $8^{\circ}$ de E.G.B. y que cursaban dichos estudios en colegios subvencionados situados en la ciudad de Córdoba.

\section{Instrumentos de medida}

La evaluación de la variable depresión se ha realizado a través del Cuestionario de Depresión Infantil (Children's Depression Inventory, CDI-S; Kovacs, 1992) en su forma corta o abreviada, cuya adapta- 
ción española ha sido realizada por Del Barrio et al. (1997).

La evaluación de la ansiedad estado y rasgo, se llevó a cabo mediante el Cuestionario de Autoevaluación de Ansiedad (State-trait Anxiety Inventory for Children, STAIC E/R) de Spielberger et al. (1973), cuya traducción y baremación en España se llevo a cabo por Seisdedos (1990).

Para realizar la valoración de la autoestima, recurrimos a la Escala de Autoestima Global (Global Self-Esteem Scale, GSES) creada por Rosenberg (1965) en su adaptación española (Del Barrio, 1994).

Con el fin de evaluar las habilidades sociales se utilizó el Autoinforme sobre Comportamiento Social de Michelson y Wood (1982).

La valoración de las habilidades en solución de problemas, se llevó a cabo mediante el cuestionario SPP-A (Cuestionario de Habilidades de Solución de Problemas Personales para Adolescentes) de reciente creación y baremación en población española (Olmedo, Santed, Fernández y Valiente, 1997).

\section{Diseño metodológico}

Con el fin de mostrar objetivamente el nivel de eficacia del programa, recurrimos a la utilización de un diseño cuasi-experimental con grupo de control no equivalente (de edad, género y características sociales análogas). Este diiseño, según las características del presente trabajo, se podría considerar la estrategia óptima de investigación (Forns, 1995; Tomlinson, Bland y Moon, 1993), ya que proporcionaría información clara, objetiva y fiable, acerca de los efectos que el programa en general y sus distintos componentes en particular, produce en los niveles de depresión y ansiedad en la adolescencia.

La estrategia metodológica se configura a partir de dos grupos, uno al que se aplica el programa o tratamiento y otro grupo al que no (la imposibilidad de seleccionar a los sujetos de forma aleatoria es la que impide que se trate de un diseño experimental). A pesar de ello, se podría alegar que los datos que se recogen proviamente, antes de la implantación del programa, permiten determinar si los alumnos de ambos grupos son similares en las características referentes a la salud mental así como en otras características que puedan resultar relevantes, en cuyo caso se podría tener cierta confianza en que las diferencias que se observen en la prueba posterior, en lo que se refiere a salud mental, serían resultado del programa.

\section{Procedimiento}

Una vez seleccionadas las variables de intervención que iban a ser objeto de nuestro programa, se procedió a realizar un plan pormenorizado para llevarlo a la práctica. Dicho plan, además del diseño clásico aludido, incluye unas determinadas secuencias de actuación que pasamos a describir siguiendo la línea temporal en la que se llevaron a cabo.

Se seleccionaron los grupos (de intervención y comparación) en función de las características relativas al nivel socio-económico, sexo, rendimiento académico y nivel de cooperación que, los directores y profesores de los colegios, estaban dispuestos a asumir en la realización de nuestro programa. Posteriormente, se aplicaron los cuestionarios para la obtención de la línea base. Dicha tarea la realizaron los tutores escolares, tanto en el grupo de intervención como en el de comparación, bajo instrucciones especí icas. La decisión de que fuesen los tutores los encargados de pasar los cuestionarios de autoinforme, fue tomada bajo la presunción de que este hecho disminuiría el sesgo debido al efecto del experimentador. Con el fin de homogeneizar la toma de datos se eligió al tutor para realizar esta labor en todos los 
momentos (también realizaron, a lo largo de todo el proceso, la evaluación de las variables que formaban parte de la intervención: autoestima, ansiedad estado, habilidades sociales y solución de problemas), siendo esto así tanto para el grupo experimental como para el control.

En este punto, y antes de comenzar la intervención, también se llevaron a cabo los análisis de tipo descriptivo e inferencial de las variables estudiadas, con el fin de comprobar la homogeneidad entre el grupo control y experimental y eliminar de la muestra a aquellos sujetos que sobrepasaron el punto de corte en ansiedad y/o depresión, ofreciéndose en estos casos ayuda particularizada.

Una vez analizados los datos anteriores, pasamos a evaluar la autoestima (tarea que, como hemos mencionado anteriormente, realizaron los tutores). A partir de los datos obtenidos en dicha variable realizamos dos tipos de análisis, el primero de ellos dirigido, como en la anterior ocasión, a comprobar la homogeneidad existente intergrupo, mientras que el segundo tenía como finalidad investigar la relación entre dicha variable y los niveles de depresión y ansiedad rasgo, justificando de esta forma su inclusión como variable sobre la que intervenir en nuestro programa.

Este proceso se realizó antes de comenzar cada uno de los cuatro módulos que componen nuestro programa, por lo cual, y antes de llevar a cabo la intervención concreta en cada uno de ellos en el grupo experimental, se realizaron los análisis de datos pertinentes en orden a comprobar empíricamente la hipótesis de que dicha variable constituía un predictor significativo de la depresión y la ansiedad, condición que consideramos previa a la puesta en práctica de la intervención planificada (véase apartado dedicado a condiciones previas).

La intervención realizada en el grupo experimental se impartió a lo largo de 18 semanas consecutivas. El tiempo dedicado a la misma fue de una hora semanal en cada una de las tres clases que componían dicho grupo.

En todo momento se contó con la colaboración tanto del equipo directivo del colegio como de los tutores, para llevar a cabo el proyecto de la forma previamente planificada.

Una semana despuěs de la última intervención en el grupo experimental, el tutor correspondiente a cada clase pasó por segunda vez los cuestionarios de depresión y ansiedad rasgo, hecho que se realizó de forma simultánea en el grupo de comparación.

Una vez recogidos estos datos, pasamos al análisis estadístico, cuya finalidad principal fue la valoración objetiva de la eficacia del programa. Para ello se realizaron diversas comparaciones entre las valoraciones obtenidas en el grupo experimental y control, dado que los cambios rápidos que aparecen entre la infancia y la adolescencia hacen que resulte imprescindible diferenciar entre los cambios puramente madurativos y aquéllos que son el resultado de una intervención dirigida.

Igualmente se realizaron diversos análisis dirigidos a dilucidar la aportación concreta que cada variable tratada en el programa había realizado al cambio obtenido en depresión y ansiedad en el grupo de intervención, una vez controlada la influencia que en este sentido podía ejercer el rendimiento académico de los sujetos.

Por último, estimamos conveniente realizar una tercera recogida de información, tanto en el grupo de intervención como en el de comparación, con el objetivo de contar con una medida de seguimiento que nos proporcionara datos acerca de la capacidad del programa para mantener los resultados obtenidos a medio plazo. Así, cinco meses después de haber finalizado la intervención, volvimos a pasar las pruebas de autoinforme sobre depresión y ansiedad rasgo. 


\section{Condiciones previas}

Antes de pasar a la exposición de los resultados que dan cuenta de los objetivos anteriormente mencionados, consideramos necesario adelantar aquéllos que fueron obtenidos en relación a dos condiciones previas que se tenían que ver cumplidas antes de la aplicación del programa tal y como este se había planeado. La primera de ellas guarda relación con el diseño metodológico y hace referencia a la equivalencia en el punto de partida de los grupos de intervención y comparación en relación a las variables relevantes para nuestro estudio. La otra condición previa se centra en la demostración de que las variables sobre las que actuamos en nuestro programa de intervención se relacionan con la depresión y la ansiedad rasgo, en nuestra muestra y con los instrumentos utilizados, dada la ausencia de trabajos anteriormente realizados de forma sistemática que se puedan considerar análogos al planteado en nuestra investigación.

El supuesto de homogeneidad se vio cumplido para todas y cada una de las variables analizadas entre las que incluimos el rendimiento escolar, no encontrándose diferencias significativas (utilizando el estadístico $t$ de Student) en ningún caso entre el grupo de intervención y el de comparación.

Respecto al segundo requisito que hemos considerado como condición previa a la valoración del programa, constatamos, antes de llevar a cabo la intervención en cada una de las variables que lo conforman (autoestima, ansiedad estado, habilidades sociales y solución de problemas), que las relaciones establecidas a nivel teórico entre ellas y la depresión y ansiedad rasgo, aparecían también en nuestra muestra.

De esta forma comprobamos la existencia de una correlación entre depresión y ansiedad rasgo que, tal como se esperaba, fue alta y significativa $(r=$ $0,62, p<0,001$ ). Igualmente, con el fin de asegurar que la intervención se realizaba sobre las variables adecuadas, comprobamos la significación del potencial explicativo que, cada una de las variables de intervención, tenía respecto a la depresión y la ansiedad rasgo, después de anular estadísticamente el efecto que, la variable rendimiento escolar y las variables que anteriormente tratadas, mantenían con dichos aspectos emocionales. Para dilucidar tal cuestión recurrimos a análisis estadísticos de regresión múltiple (método enter) (Tablas 1 y 2).

Tabla 1. Análisis de regresión múltiple jerárquica con variable(s) control, de las medidas pretest de rendimiento escolar (notas 1), de la variable criterio depresión (CDI-S 1) sobre las independientes: autoestima (GSES 1), ansiedad estado (STAIC-E 1), habilidades sociales (CABS 1) y solución de problemas (SPP-A 1)

\begin{tabular}{ccccc}
\hline $\begin{array}{c}\text { Predictor } \\
\text { (paso 2) }\end{array}$ & Autoestima & AE & HS & SP \\
\hline Control & & & & Notas 1 \\
(paso 1) & & Notas 1 & Autoestima & $\begin{array}{c}\text { Autoestima } \\
\end{array}$ \\
& Notas 1 & Autoestima & AE & HS \\
\hline$R^{2}$ cambio & $\mathbf{0 , 3 3 8}$ & 0,175 & 0,021 & 0,007 \\
$F$ cambio & 136,224 & 102,980 & 13,645 & 4,782 \\
Sig. F & 0,000 & 0,000 & 0,000 & 0,029 \\
$\beta$ & $-0,592$ & 0,462 & $-0,170$ & $-0,092$ \\
C.C. Parcial & $-0,616$ & 0,563 & $-0,240$ & $-\mathbf{0 , 1 4 6}$ \\
\hline
\end{tabular}

Nota: $\mathrm{AE}=$ Ansiedad estado, $\mathrm{HS}=$ Habilidades sociales y $\mathrm{SP}=$ Solución de problemas. 
Tabla 2. Análisis de regresión múltiple jerárquica con variable(s) control, de las medidas pretest de rendimiento escolar (notas 1), de la variable criterio ansiedad rasgo (STAIC-R 1) sobre las variables independientes: autoestima (GSES 1), ansiedad estado (STAIC-E 1), habilidades sociales (CABS 1) y solución de problemas (SPP-A 1)

\begin{tabular}{ccccc}
\hline $\begin{array}{c}\text { Predictor } \\
\text { (paso 2) }\end{array}$ & Autoestima & AE & HS & SP \\
\hline Control & & & & Notas 1 \\
(paso 1) & & Notas 1 & Autoestima & Autoestima \\
& & AE \\
& Notas 1 & Autoestima & AE & HS \\
\hline$R^{2}$ cambio & 0,149 & 0,276 & 0,016 & 0,002 \\
$F$ cambio & 39,729 & 109,360 & 6,826 & 1,054 \\
Sig. F & 0,000 & 0,000 & 0,009 & 0,305 \\
$\beta$ & $-\mathbf{0 , 3 9 4}$ & 0,580 & $-0,149$ & $-0,054$ \\
C.C. Parcial & $-\mathbf{0 , 3 8 9}$ & 0,575 & $-\mathbf{0 , 1 7 3}$ & $-0,069$ \\
\hline
\end{tabular}

Nota: $\mathrm{AE}=$ Ansiedad estado, $\mathrm{HS}=$ Habilidades sociales y $\mathrm{SP}=$ Solución de problemas.

En definitiva, podemos afirmar que las diferentes variables seleccionadas a nivel teórico resultaron ser empíricamente adecuadas para llevar a cabo nuestra intervención, hecho que permitió determinar la bondad del contenido del programa de intervención, cuyos resultados pasamos a exponer a continuación.

\section{RESULTADOS}

En lo que se refiere a la evaluación del programa propuesto, se presentarán los efectos o resultados obtenidos a través de su implantación, de los cuales podemos destacar algunas cuestiones que hemos considerado relevantes.

Siguiendo el diseño cuasi-experimental que hemos elegido para realizar dicha valoración, resulta fundamental la realización de cuatro grupos de comparaciones (mediante el estadístico $t$ de Student) El primer grupo de ellas hace referencia a las comparaciones de las puntuaciones en línea base, tanto en las variables dependientes (depresión y ansiedad rasgo), como en las variables sobre las que realizamos la intervención (autoestima, ansiedad estado, habilidades sociales y solución de problemas), entre el grupo de intervención y control, con la finalidad de comprobar la homogeneidad entre los mismos. Como ya se ha dicho con anterioridad en el apartado de condiciones previas, los resultados de dichas comparaciones no reflejaron diferencias significativas en ningún caso.

Un segundo grupo de comparaciones engloba los contrastes de medias realizados en el grupo de intervención, tanto para las variables de intervención, antes y después del tratamiento en cada una de ellas, como para las variables dependientes (depresión y ansiedad rasgo) en línea base y una vez finalizado todo el programa. A través de la observación de los resultados encontrados en este sentido, podemos afirmar que el grupo experimental presentó cambios en la dirección esperada después de la intervención, que resultaron ser estadísticamente significativos.

En lo que se refiere a la variable depresión (CDI-S) se encontró que los niveles descendieron significativamente desde el momento anterior a la intervención hasta la medida tomada con posterioridad a la realización del tratamiento $[M=3,68$ y $D T=2,27$ en línea base, y $M=2,79$ y $D T=$ 1,18 en el segundo momento; $t(120)=$ $4,32, p<0,001$ ].

Igualmente, la ansiedad rasgo evaluada a través del STAIC-R presentó un 
decremento en la medida tomada después de concluir la intervención [ $M=$ 36,09 y $D T=6,31$ en el momento de línea base y $M=34,00$ y $D T=7,4$ en el segundo momento; $t(120)=3,93, p<0,001]$, confirmándose también, en este caso, la existencia de diferencias significativas entre ambas medidas.

En cuanto al cambio producido por cada una de las técnicas enseñadas dirigidas a mejorar la autoestima, la ansiedad estado, las habilidades sociales y la solución de problemas, podemos decir que los módulos implantados mejoraron significativamente los niveles de dichas variables, alcanzado de esta forma su objetivo. Los resultados hallados en este sentido se exponen a continuación.

Para la variable autoestima se encontraron diferencias significativas entre las medidas tomadas en línea base y al finalizar la intervención $[M=32,19$ y $D T=2,9$ y $M=34,52$ y $D T=2,8$, respectivamente; $t$ $(120)=-10,47, p<0,001]$, lo que muestra un incremento de la autoestima en este grupo. Sin embargo, en la variable ansiedad estado no se confirmó la existencia de diferencias significativas entre los momentos anterior y posterior al tratamiento $[M=29,93$ y $D T=6,8$ en línea base y $M=29,24$ y $D T=7,8$ al finalizar la intervención; $t(120)=0,93, p=0,35$ ], ya que, como podemos observar, se aprecia un ligero descenso que no llega a alcanzar niveles significativos. Respecto a las medidas tomadas en habilidades sociales (CABS) en los momentos mencionados podemos confirmar, de nuevo, diferencias significativas entre ellas. La media del porcentaje de respuestas correctas totales en habilidades sociales fue, en el caso de la medida pretest, 68,64 ( $D T=$ $12,08)$ y para la medida postest $M=76,49$ y $D T=15,50 ; t(120)=-5,13, p<0,001$. Por último, la comparación realizada entre ambos momentos en la variable solución de problemas (SPS-A), también reflejó cambios significativos en el número de respuestas correctas alcanzadas: $M=4,09$ y $D T=2,2$, en línea base, y $M=6,59$ y $D T=2,08$, para la medida tomada al finalizar la iniervención; $t(120)=-11,17, p<$ 0,001 .

En resumen, a través de la observación de los resultados expuestos podemos afirmar que el grupo experimental presentó cambios en la dirección esperada después de la intervención. Estos resultaron ser estadísticamente significativos en todas las variables evaluadas, salvo en el caso de la ansiedad estado, en la que sólo se manifestó una tendencia al decremento que no llegó a ser significativa.

Ahora bien, dichos cambios podrían atribuirse tanto al efecto del tratamiento como al paso del tiempo $u$ otras variables extrañas. Por este motivo recurrimos a un tercer grupo de comparaciones, realizado esta vez entre los datos obtenidos en el grupo de control en los momentos 1 y 2 , que nos proporciona información sobre el efecto que el paso del tiempo puede tener en las variables evaluadas. A modo de resumen, puede decirse que el grupo de comparación ha permanecido estable a lo largo del tiempo en cuanto a las variables de depresión, ansiedad rasgo, respuestas correctas en habilidades sociales y solución de problemas. Sin embargo, las variables de autoestima y ansiedad estado presentaron cambios que indican un deterioro; así, la autoestima decreció con el tiempo $[M=32,50, D T=2,9$ en línea base y $M=32,01, D T=3,02$ en el postest; $t(103)=2,35, p=0,021]$ y la ansiedad estado aumentó $[M=30,11, D T=$ 5,79 en el primer momento y $M=31,30$, $D T=6,30$ en el segundo; $t(103)=-2,53$, $p=0,013$.

Un último grupo de comparaciones realizadas, en esta ocasión, con la finalidad de contrastar los resultados entre el grupo de intervención y el de control en el momento 2 (una vez finalizado el tratamiento en el primero) permite atribuir las diferencias existentes entre las pun- 
tuaciones obtenidas en las variables evaluadas, al efecto del tratamiento, puesto que se supone que el tiempo y las posibles variables extrañas actúan en ambos grupos. Así pues, comprobamos que en la medida de depresión (CDI-S), hay diferencias significativas entre los grupos de intervención y comparación, mostrando esta variable unos niveles menores en el primero respecto al segundo $[M=2,79$, $D T=1,18$ y $M=3,58, D T=2,10$, respectivamente; $t(223)=-3,00, p=0,003]$.

La ansiedad rasgo evaluada a través del STAIC-R presentó, en el retest, los siguientes valores: $M=34$ y $D T=7,4$ para el grupo de intervención y $M=36,62$ y $D T=6,37$ para el grupo de comparación, $t(223)=-2,83 p=0,005$, confirmándose en este caso, igualmente, la existencia de diferencias significativas entre ambos grupos.

En la variable autoestima (GSES), se encontraron diferencias significativas entre las medidas tomadas en el grupo de intervención y control al finalizar el tratamiento: $M=34,52(D T=2,8)$ en el grupo de intervención, y $M=32,01(D T=3,02)$ en el grupo control; $t(223)=6,41, p<$ 0,001 .

En la variable ansiedad estado (STAICS) se confirmó igualmente la existencia de diferencias significativas entre los grupos de intervención y comparación, en el momento posterior al tratamiento $[M=$ 29,24 y $D T=7,8$ en el grupo de intervención y $M=31,30$ y $D T=6,3$ en el grupo de comparación; $t(223)=-2,15, p=0,032]$.

Respecto a las medidas tomadas en habilidades sociales (CABS) la media del porcentaje de respuestas correctas totales fue 76,49 $(D T=15,50)$ en el caso del grupo en el que se realizó la intervención y para el grupo control $M=69,11$ (DT= 11,85); $t(223)=3,96, p<0,001$.

Por último, para la variable solución de problemas, los resultados respecto a las respuestas correctas fueron los siguientes: $M=6,59(D T=2,08)$ en el gru- po de intervención y $M=4,67(D T=1,58)$ en el grupo de comparación $[t(223)=$ $7,83, p<0,001]$, reflejándose, una vez más, diferencias significativas en situación retest entre ambos grupos.

Como hemos podido observar, los datos apoyan la tesis de la eficacia del tratamiento, lo que permite suponer que nuestro programa ha conseguido obtener en el grupo de intervención los efectos pretendidos.

Llegados a este punto, en que se ha puesto de manifiesto la efectividad del tratamiento realizado, cabría preguntarse cómo podría mejorarse la intervención realizada.

Para conocer las posibles mejoras hemos determinado, a partir de los resultados obtenidos, el mejor paquete integrado de técnicas de intervención (modelo explicativo-predictivo) dirigido a la disminución de los niveles de depresión y ansiedad rasgo. Con esta finalidad recurrimos al análisis estadístico de regresión múltiple, método stepwise. Tomando el cambio en depresión (CDI-S momento 2 - CDI-S momento 1) como variable criterio, y el cambio en el conjunto de variables que forman el paquete terapéutico como predictoras, los resultados obtenidos mediante regresión múltiple figuran en la Tabla 3.

El modelo incluyó la ansiedad estado en primer lugar ( $R^{2}$ ajustada=0,221), es decir, mediante la puntuación obtenida en el cambio de ansiedad estado antes y después de la intervención en relajación, podemos predecir un $22 \%$ de la varianza producida en el cambio en depresión. En segundo lugar, el análisis stepwise introdujo la variable cambio en autoestima, aumentando la capacidad predictiva del modelo hasta explicar mas del $28 \%$ de la varianza en el cambio obtenido en depresión $\left(R^{2}\right.$ ajustada=0,284). Por último, al agregar al modelo la variable de cambio en solución de problemas la predicción en la variable criterio aumento hasta 
alcanzar casi el $32 \%$ de predicción de la varianza $\left(R^{2}\right.$ ajustada $\left.=0,318\right)$.

Sin embargo, el cambio obtenido a través del módulo de habilidades sociales no resultó ser un factor capaz de contribuir significativamente, dentro de este modelo, a la explicación de los niveles de varianza obtenidos en el cambio de la depresión.

Después de haber analizado la contribución aportada por los diferentes módulos que conforman el programa a la hora de disminuir los niveles de depresión en el grupo experimental, pasamos a repetir el mismo procedimiento, tomando esta vez como variable criterio el cambio en ansiedad rasgo, con el fin de comprobar qué estrategias de intervención obtenían un poder predictivo significativo respecto al cambio en la misma.

Los resultados que nos aporta el análisis de regresión múltiple realizado figuran en la Tabla 4. Como puede ubservarse, el cambio en autoestima y en solución de problemas son dos variables no incluidas en el modelo predictivo, ya que no explicaron una proporción de varianza significativa de forma añadida a la que en su conjunto eran capaces de explicar el cambio en ansiedad estado y en habilidades sociales. Estas dos últimas variables, que sí entraron a formar parte de la ecuación de regresión, eran capaces de explicar, en su conjunto, un $49 \%$ de la varianza correspondiente al cambio en ansiedad rasgo.

Tabla 3. Análisis de regresión múltiple (ARM) del criterio cambio en depresión (CDI-S 2 - CDI-S 1) sobre las variables independientes: cambio en ansiedad estado, cambio en autoestima, cambio en habilidades sociales y cambio en solución de problemas

\begin{tabular}{ccccc}
\hline $\begin{array}{c}\text { Estadísticos } \\
\text { ARM }\end{array}$ & Ansiedad estado & Autoestima & $\begin{array}{c}\text { Solución de } \\
\text { problemas }\end{array}$ & $\begin{array}{c}\text { Habilidades } \\
\text { sociales }\end{array}$ \\
\hline$R$ Múltiple & 0,477 & 0,532 & 0,579 & \\
$R^{2}$ & 0,228 & 0,284 & 0,335 & \\
$R^{2}$ ajustada & 0,221 & 0,271 & 0,318 & \\
$F$ & 35,091 & 23,353 & 19,642 & \\
Sig. $F$ & 0,000 & 0,000 & 0,000 & $0,052 \mathrm{n} / \mathrm{s}$ \\
$\beta$ & 0,396 & $-0,236$ & $-0,233$ & \\
\hline
\end{tabular}

Nota: Las variables independientes han sido operativizadas de la siguiente forma: Ansiedad estado = ansiedad estado Momento 2 - ansiedad estado Momento 1. Autoestima = autoestima Momento 2 - autoestima Momento 1. Solución de problemas $=$ solución de problemas Momento 2 - solución de problemas Momento 1. Habilidades sociales $=$ habilidades sociales Momento 2 - habilidades sociales Momento 1.

Tabla 4. Análisis de regresión múltiple (ARM) del criterio cambio en ansiedad rasgo (STAIC-R 2-STAIC-R 1), sobre las variables independientes: cambio en ansiedad estado, cambio en autoestima, cambio en habilidades sociales y cambio en solución de problemas

\begin{tabular}{ccccc}
\hline $\begin{array}{c}\text { Estadísticos } \\
\text { ARM }\end{array}$ & Ansiedad estado & Autoestima & $\begin{array}{c}\text { Solución de } \\
\text { problemas }\end{array}$ & $\begin{array}{c}\text { Habilidades } \\
\text { sociales }\end{array}$ \\
\hline$R$. Múltiple & $\mathbf{0 , 3 9 6}$ & 0,489 & & \\
$R^{2}$ & $\mathbf{0 , 1 5 7}$ & 0,239 & & \\
$R^{2}$ ajustada & $\mathbf{0 , 1 5 0}$ & 0,226 & & \\
$F$ & 22,166 & 18,574 & & \\
Sig. $F$ & $\mathbf{0 , 0 0 0}$ & 0,000 & $0,577 \mathrm{n} / \mathrm{s}$ & $\mathbf{0 , 5 7 3} \mathrm{n} / \mathrm{s}$ \\
$\beta$ & $\mathbf{0 , 3 3 6}$ & 0,293 & & \\
\hline
\end{tabular}

Nota: Las variables independientes han sido operativizadas de la siguiente forma: Ansiedad estado = ansiedad estado Momento 2 - ansiedad estado Momento 1. Habilidades sociales = habilidades sociales Momento 2 - habilidades sociales Momento 1 . Autoestima = autoestima Momento 2 - autoestima Momento 1 . Solución de problemas $=$ solución de problemas Momento 2 - solución de problemas Momento 1. 
La observación de tales datos y su comparación con los obtenidos tomando como variable criterio el cambio en depresión, nos puede llevar a afirmar que el paquete terapéutico empleado es más adecuado para disminuir los niveles de depresión que los de ansiedad, ya que explica, conjuntamente, una proporción mayor de la variabilidad obtenida en el cambio de la primera variable. Sin embargo, el hecho de que el cambio en habilidades sociales haya resultado, respecto a la ansiedad rasgo, una variable predictiva de eficacia, aspecto que no se cumple en la depresión, hace que podamos considerar todos estos módulos de intervención como complementarios, aceptando de esta forma la bondad de nuestro paquete integrado de técnicas como predictor para lograr efectos de cambio tanto en depresión como en ansiedad rasgo.

Finalmente, estimamos conveniente obtener información respecto a la capacidad del programa para mantener sus efectos una vez concluido el tratamiento. Para ello, se realizó una medida de seguimiento tanto en el grupo experimental como en el de control a los cinco meses de haber finalizado nuestra intervención en el primero.

El análisis de datos se llevó a cabo, al igual que en anteriores ocasiones, a través de diferentes comparaciones utilizando el estadístico $t$. En la primera de ellas analizamos las diferencias obtenidas en el grupo experimental entre la línea base (momento 1) y los datos procedentes de la valoración de seguimiento (momento 3), respecto a la variable depresión (CDI-S). Concretamente, encontramos cambios significativos entre ambos momentos, mostrando esta variable un decremento respecto a la primera en la medida de seguimiento $[M=3,68$ y $D T=2,27$ en línea base, y $M=3,02$ y $D T=$ 1,66 en el seguimiento (momento 3 ); $t$ $(120)=2,95, p=0,004]$.
Con la finalidad de determinar la estabilidad de los efectos del programa, el segundo contraste de medias realizado aporta información acerca de las diferencias entre las medidas obtenidas una vez concluida la intervención (momento 2) y la medida de seguimiento (momento 3 ) en el grupo experimental. Los resultados obtenidos en la variable depresión son: $M=2,79$ y $D T=1,81$ en el momento $2, \mathrm{y}$ $M=3,02$ y $D T=1,66$ en el seguimiento (momento 3); $t(120)=-1,31, p=0,193$ ). Así pues, los niveles de depresión se mantuvieron sin cambios significativos durante cinco meses.

Las mismas comparaciones, realizadas esta vez en relación a los datos obtenidos en la variable ansiedad rasgo, evaluada a través del STAIC-R, presentaron igualmente diferencias significativas entre los momentos 1 y 3 en el grupo experimental, cuyas medias y desviaciones típicas fueron $M=36,09$ y $D T=6,31$ y $M=34,46$ y $D T=6,55$, respectivamente, $t(120)=$ $3,12, p=0,002$. Por otra parte, al igual que ocurría con la variable depresión, las comparaciones entre los datos obtenidos en los momentos 2 y 3 no reflejaron diferencias significativas $[M=34,00$ y $D T=$ 7,40 y $M=34,46$ y $D T=6,55$, respectivamente, $t(120)=-1,47, p=0,143]$. Como en ambos casos no hemos encontrado diferencias significativas, se puede asumir que los niveles de depresión y ansiedad en ambos momentos son equiparables, lo que demuestra la permanencia de la eficacia del programa.

A partir de los anteriores resultados podríamos afirmar que los efectos del programa se mantienen pasado un periodo de tiempo de cinco meses desde que concluimos la intervención, aunque en este punto cabría interpretar que dichos resultados pueden deberse a otros factores como pueden ser el paso del tiempo y la maduración de los sujetos, o el hecho de pasar por tercera vez los mismos cuestionarios. Para controlar esta 
posibilidad, repetimos el proceso de análisis de datos anterior, esta vez tomando como referencia el grupo control. Seguidamente pasamos a exponer los resultados que obtuvimos en este sentido.

En el grupo de control no aparecieron diferencias de medias significativas en la medida de depresión (CDI-S) entre los momentos de línea base $(M=3,47$ y $D T=$ 2,32) (momento 1) y el correspondiente al seguimiento (momento 3$)(M=3,55 \mathrm{y}$ $D T=1,99), t(103)=-0,37, p<0,714$. Igualmente, los contrastes de medias realizados entre los momentos 2 y 3 resultaron, para este grupo, no significativos: $M=$ 3,58 y $D T=2,10$ en el momento 2 , y $M=$ 3,55 y $D T=1,99$ en el seguimiento (momento 3); $t(103)=0,16, p=0,874$.

Respecto a la variable ansiedad rasgo (STAIC-R), el grupo control tampoco manifestó diferencias significativas entre el momento 1 y $3: M=35,91, D T=6,97$ en el momento 1 ; y $M=36,86, D T=5,82$ en el momento 3 ; $t(103)=-1,40, p=0,166$. Tampoco entre los momentos 2 y $3: M=$ 36,62 y $D T=6,37$ y $M=36,86$ y $D T=5,82$, respectivamente, $t(103)=0,43 p=0,670$.

Estos resultados ponen de manifiesto el escaso efecto que, en ausencia de intervención, tiene el paso del tiempo o el pase repetitivo de los cuestionarios en las variables estudiadas considerado un periodo de cinco meses. Una vez conocido este hecho y con la finalidad de afianzar la valoración del programa en cuanto a la duración de sus efectos, pasamos finalmente a presentar los resultados de la comparación entre el grupo experimental y control en el momento 3 (medida de seguimiento), y que reflejan que las diferencias halladas después de realizar la intervención en el grupo experimental continúan apareciendo.

En la medida de depresión (CDI-S) se confirmó la existencia de diferencias entre ambos grupos en la medida de seguimiento, presentando el grupo experimental unas puntuaciones con $M=3,02$ y $D T=1,66$, y el grupo control $M=3,55$ y $D T=1,99 ; t(223)=-2,16, p=0,032$.

Las puntuaciones en la medida de ansiedad rasgo (STAIC-R) en el caso del grupo experimental fueron: $M=34,46$ y $D T=6,55$ y para el control $M=36,87$ y $D T=5,82 ; t(223)=-2,89, p=0,004$, confirmándose en este caso, igualmente, la existencia de una diferencia significativa entre ambos grupos en una dirección que apoyaría la hipótesis de que los efectos de nuestro programa se mantienen a medio plazo.

Como vemos, las diferencias en depresión y ansiedad rasgo entre el grupo experimental y control, obtenidas al finalizar el tratamiento, se mantienen, no regresando a la homogeneidad que, en tales variables, hallamos en línea base entre ambos grupos.

\section{DISCUSIÓN}

A partir de los resultados expuestos podemos destacar algunas cuestiones que hemos considerado relevantes. En primer lugar, cabe decir que las variables seleccionadas para conformar el programa son adecuadas para lograr la meta planteada. De esta forma podríamos concluir de acuerdo con Garber, Weiss y Shanley (1993) que es posible actuar de manera conjunta para disminuir los niveles de ambas variables (depresión y ansiedad) mediante estrategias de intervención orientadas a mejorar los déficits existentes en los factores mencionados.

Los resultados obtenidos a través de las diferentes comparaciones que propicia el diseño cuasi-experimental empleado en nuestra investigación, nos llevan a considerar que la aplicación de nuestro programa de intervención ha tenido los efectos deseados, es decir, que hemos logrado disminuir los niveles de depresión y ansiedad en la población adolescente a la que ha sido aplicado, cum- 
pliéndose de esta forma el objetivo principal de nuestro trabajo.

Una cuestión pendiente sería demostrar la generalización de estos resultados, cuestión cuya respuesta necesitaría de más aplicaciones del programa en otras poblaciones.

Hasta aquí, los resultados obtenidos por nuestra investigación señalan los efectos beneficiosos del programa implantado, sin embargo la evaluación de programas, además de requerir la emisión de juicios sobre la efectividad del tratamiento realizado, presenta otra característica básica: es un recurso para la mejora de las acciones que, debidamente organizadas, se implantan en un determinado contexto social (Fernández-Ballesteros, 1995). Visto nuestro programa bajo esta perspectiva cabría preguntarse cómo podría mejorarse la intervención realizada, y qué debilidad encontrada es susceptible de modificación.

Para responder a estas cuestiones, hubiese sido de gran ayuda contar con investigaciones previas que, realizadas en el ámbito escolar, hubiesen llevado a cabo intervenciones semejantes a la aplicada en nuestro caso, con el fin de comparar si los resultados cambian y en función de qué factores lo hacen. Sin embargo, la valoración de nuestra intervención cuenta con una limitación en este sentido, dado que no hemos encontrado un trabajo que, de forma empírica y generalizable a nuestra población, esté dedicado a indagar comparativamente la eficacia de los diferentes tratamientos que hemos utilizado en nuestro programa de intervención.

En este punto cabe señalar que la mayoría de los trabajos que presentan una descripción más sistemática de las estrategias de entrenamiento utilizadas suelen estar centrados en una única variable como como puede ser la autoestima (Mestre y Frías, 1996), las habilidades sociales (Caballo, 1987; Michelson, Sugai, Wood, y Kazdin, 1984); la solu- ción de problemas (Pelechano, 1996), o la ansiedad estado (Cautela, y Groden, 1985), siendo imposible realizar una comparación entre los resultados obtenidos debido a la diferencia de las muestras empleadas en los mismos.

Dadas las condiciones mencionadas en los estudios que podrían ayudarnos a la realización de comparaciones dirigidas a mejorar nuestro programa, debemos centrarnos en la información que los análisis estadísticos, realizados a partir de los datos obtenidos en el grupo experimental de nuestra muestra, nos proporcionan. En este sentido, mediante el análisis de regresión análisis múltiple (stepwise) tratamos de determinar el mejor paquete integrado de técnicas para disminuir los niveles de depresión y ansiedad rasgo en nuestra muestra de adolescentes.

Del conjunto de datos expuestos con anterioridad en relación a este objetivo podemos destacar tres observaciones. En primer lugar, el hecho de que el incremento conseguido a través de la intervención en la variable habilidades sociales no resultara un factor capaz de contribuir significativamente a la explicación de los niveles de varianza obtenidos en el cambio en depresión, es un hallazgo en cierta medida incongruente con las afirmaciones realizadas por diversos autores respecto a los beneficios que el entrenamiento en habilidades sociales supone de cara a la mejora de la depresión. Por ejemplo, Groosman y Hughes (1992) realizaron una revisión de trabajos que habían empleado este tipo de recursos, concluyendo que el entrenamiento en habilidades sociales resultaba especialmente eficaz para tratar problemas de carácter interiorizado en adolescentes. Un posible argumento para explicar la falta de concordancia entre las anteriores afirmaciones y los resultados de nuestro estudio, podría basarse en el hecho de que la intervención en habilidades sociales resulta beneficiosa en el caso de tra- 
tarse de sujetos con problemas emocionales, en los que el nivel de habilidades sociales se encuentra deteriorado (Beck, 1983; Seligman, 1975; Shaffi y Shaffi, 1992), pero no así cuando se trata de disminuir los niveles de sintomatología depresiva en población normal, en cuyo caso, al margen de confirmarse la relación existente entre ambas variables (Cole y Carpentieri, 1990; Kazdin et al., 1985; Lewinsonh et al., 1994), el incremento de habilidades sociales no se corresponde exactamente con un decremento en depresión. Por tanto, de cara a futuras implantaciones del programa, podríamos aconsejar prescindir del módulo de habilidades sociales en el contenido del mismo cuando la finalidad de la intervención este centrada exclusivamente en la disminución de los niveles de depresión, ya que, según los datos obtenidos en nuestro estudio, los resultados conseguidos a través de dicha intervención no han influido significativamente en la reducción de los niveles de depresión.

Una segunda observación, que podemos considerar relevante, hace referencia al hecho de que la capacidad predictiva de nuestro programa, respecto a la disminución de la ansiedad, se cierra con los módulos dedicados a la relajación y a las habilidades sociales. Los resultados obtenidos mediante las estrategias de intervención restantes, empleadas para mejorar la autoestima y la capacidad de solución de problemas, no aportaban explicación al cambio en ansiedad. Así pues, cuando la realización de un programa de intervención en población no clínica adolescente tenga como única finalidad disminuir los niveles de ansiedad en los sujetos tratados, los resultados aportados por nuestro estudio aconsejarían realizar exclusivamente los módulos dedicados al incremento de habilidades sociales y relajación, siendo posible suprimir, en este caso, los dedicados a elevar la autoestima y la capacidad de solución de problemas.

Finalmente, podríamos concluir que el contenido de nuestro programa se puede considerar adecuado cuando el objetivo de la intervención sea disminuir, de forma conjunta, los niveles de depresión y ansiedad en población no clínica adolescente, ya que los resultados obtenidos en cada uno de los cuatro módulos que conforman el programa, aportan una explicación estadísticamente significativa en el beneficio adquirido en relación a las citadas variables. En resumen, la valoración realizada respecto al contenido de nuestro programa ha puesto de manifiesto que las distintas estrategias de intervención realizadas han contribuido diferencialmente al cambio en los niveles de depresión y ansiedad rasgo; además, podemos decir que, tomado en su conjunto, el paquete terapéutico empleado ha resultado eficaz para conseguir los objetivos propuestos, ya que las diferentes técnicas empleadas resultan complementarias de cara a de disminuir los niveles de depresión y ansiedad.

En cualquier caso, sería deseable que, antes de poner en práctica las anteriores observaciones, se confirmasen los resultados obtenidos mediante nuestra intervención en posteriores investigaciones que, sin variar el contenido del programa, utilizaran diferentes muestras, diferentes instrumentos de evaluación y diferentes entrenadores, con el fin de comprobar si bajo estas distintas condiciones pueden seguir manteniéndose las afirmaciones realizadas.

Por último, una vez mostrada la eficacia del programa para disminuir los niveles de depresión y ansiedad al término de la intervención realizada, hemos presentado datos relativos a la capacidad del programa para mantener sus consecuencias a través del tiempo. En este sentido, los resultados reflejan que los efectos 
beneficiosos conseguidos se mantenían cinco meses después de concluir la intervención. Sin embargo, en este sentido, también es posible observar una tendencia que atenúa los efectos conseguidos en principio por el tratamiento. El incremento, no significativo, de los niveles de depresión y ansiedad, hace pensar en la posibilidad de que los beneficios de nuestro programa vayan perdiendo fuerza con el paso del tiempo, en cuyo caso, sería de esperar que si hubiésemos continuado tomando medidas en momentos posteriores, dilatando el periodo de seguimiento, llegaría un momento en que los efectos de la intervención apenas se harían manifiestos.

Teniendo en cuenta la relevancia de esta posibilidad, dado que no podemos considerar la utilidad de un programa de intervención cuyos efectos son efímeros, sería conveniente, de cara a futuras investigaciones, profundizar en mayor medida en este aspecto, realizando un mayor número de evaluaciones en el periodo de seguimiento, sin desechar la posibilidad de incluir nuevos módulos de intervención orientados a los mismos objetivos, en cursos posteriores.

En definitiva, y enlazando con la idea de realizar nuevas implantaciones de programas dirigidos a la prevención primaria de problemas emocionales en la adolescencia, podemos concluir que la facilitación en el ámbito escolar del aprendizaje de estrategias que capaciten a los alumnos a enfrentarse con mayor efectividad ante las diferentes situaciones de estrés durante su crecimiento, y la enseñanza de recursos que les proporcionen un mejor conocimiento de sí mismos y un manejo más flexible y cómodo del mundo que les rodea, será siempre una labor complementaria a realizar por aquellos profesionales de la educación que estén interesados en preparar a la juventud a funcionar efectivamente a nivel emocional y social.

\section{REFERENCIAS BIBLIOGRÁFICAS}

Beck, A.T. (1983). Cognitive therapy of depression: new perspectives. En P. Clayton y J.E. Barret (Eds.), Treatment of depression. Old controversies and new approaches. Raven Press: New York.

Brandy, E. y Kendall, Ph. (1992). Comorbidity of anxiety and depression in children and adolescent. Psychological Bulleting, 111, 244-255.

Butler, L., Miezitis, S., Frieman, R. y Cole, E. (1980). The effect of two school-based intervention programs on depressive symptoms in preadolescents. American Educational Research Journal, 111-119.

Caballo, V.E. (1987). Teoría, evaluación y entrenamiento en las habilidades sociales. Valencia: Promolibro.

Cautela, J.R. y Groden, J. (1985). Técnicas de relajación, manual práctico para adultos, niños y educación especial. Barcelona: Martínez-Roca.

Cole, D.A. y Carpentieri, S. (1990). Social status and the comorbidity of child depression and conduct disorders. Journal of Consulting and Clinical Psychology, 58, 747-757.

Del Barrio, V. (1990). Aspectos socioculturales de la depresión infantil. En E. Doménech y A. Polaino (Eds.), Epidemiología de la Depresión Infantil. Barcelona: Espaxs.

Del Barrio, V. (1994). Adaptación española de la Escala de Autoestima de Rosenberg (trabajo no publicado).

Del Barrio, V. (1997). Depresión Infantil: causas, evaluación y tratamiento. Ariel: Barcelona.

Del Barrio, V., Frías, D. y Mestre, V. (1994). Autestima y depresión en niños. Revista de Psicología General y Aplicada, 47, 471-476.

Del Barrio, V., Roa, L., Olmedo, M. y Colodrón; F. (1997). Spanish adaptation of Short Children's Depression Inventory (CDI-S). Comunicación presēntáda al Regional Congress of Psychology for Professionals in the Americas. México, $27 \mathrm{de}$ julio al 2 de agosto.

Fergusson, D.M., Horwood, L.J. y Lynskey, M.T. (1995). Maternal depressive symptoms and depressive symptoms in adoles- 
cents. Journal of Child Psychology and Psychiatry, 36, 1161-1178.

Fernández-Ballesteros, R. (1995). Valoración de Programas. Madrid: Síntesis.

Forns, M. (1995). Evaluación de programas educativos. En R. Fernández-Ballesteros (Ed.), Evaluación de Programas. Madrid: Síntesis.

Garber, J., Weiss, B. y Shanley, N. (1993). Cognitions, depressive symptoms and development in adolescents. Journal of Abnormal Psychology, 102, 47-57.

Gröer, M.W., Thomas, S.P. y Shoffner (1992). Adolescent Stress and Coping: A longitudinal study. Research in Nursing $\&$ Health, 15, 209-217.

Grossman, P.B. y Hughes, J.N. (1992). Selfcontrol interventions with internalizing disorders: A review and analysis. School Psychology Review, 21, 229-245.

Kandel, D.B. y Davies, M. (1986). Adult sequelae of adolescent depressive symptoms. Archives of General Psychiatry, 43, 255-262.

Kazdin, A.E., Sherick, R.B, Esvellt-Dawson, K. y Colbus, M.D. (1985). Assessment of overt behavior and chilhood depression among psychiatrically disturbed inpatient children. Journal of Abnormal Child Psychology, 53, 201-210.

Kovacs, M. (1992). Children's Depression Inventory, CDI. Toronto: Multi-Health Systems, Inc.

Lefkowitz, M.M. y Tesiny, E.P. (1981). Assessment of childhood depression. Journal of Consulting and Clinical Psychology, 48, 43-50.

Lewinsohn, P.M., Roberts, R.E., Seeley, J.R., Rohde, P., Gotlib, I.H. y Hops, H. (1994). Adolescent psychopathology: II. Psychosocial risk factors for depression. Journal of Abnormal Psychology, 103, 302-315.

McKay, M., Davis, M. y Fanning, P. (1981). Thoughts \& feeling: The art of cognitive stress intervention. Oakland, California: New Harbinger Publications.

Mestre, V. (1992). La depresión en población adolescente valenciana. Valencia: Consellería de Sanitat i Consum.

Mestre, V. y Frías, M.D. (1996). La mejora de la autoestima en el aula. Aplicación de un programa para niños en edad escolar (11-
14 años). Revista de Psicología General y Aplicada, 49, 279-290.

Michelson, L., Sugai, D.P., Wood, R.P. y Kazdin A.E. (1984). Las Habilidades Sociales en la Infancia; Evaluación y Tratamiento. Manual práctico. Barcelona: MartínezRoca.

Michelson, L. y Wood, R. (1982). Behavioral assessment and training of children's social skills. En M. Hersen, P. M. Miller y R.M. Eisler (Eds.), Progress in behavior modifications (Vol. 9). New York: Academy Press.

Olmedo, M., Del Barrio, V. y Santed, M.A. (en prensa). Programa de afrontamiento de problemas emocionales en adolescentes.

Olmedo, M., Santed, M.A, Fernández, E. y Valiente, R.M. (1997). Habilidades de solución de problemas sociales y su relación con la depresión en la adolescencia. Presentado al I Congreso de la Asociación Española de Psicología Clínica y Psicopatología. Madrid, 3-5 de abril.

Oster, G.D. y Caro, J.E. (1990). Understanding and treating depressed adolescents and their families. New York: Wiley.

Pelechano, V. (1996). Habilidades Interpersonales: Teoría mínima y programas de intervención. (2 Vols.). Valencia: Promolibro, Alfaplus.

Polaino-Lorente, A. (1987). Las depresiones Infantiles. Madrid: Morata.

Reinherz, H.Z., Frost, A.K y Pakiz, B. (1991). Changing faces: correlates of depressive symptoms in late adolescence. Family and Community Health, 14, 52-63.

Reynolds, W.M. y Coats, K.I. (1986). A comparison of cognitive-behavior therapy and relaxation training for the treatment of depression in adolescents. Journal of Consulting and Clinical Psychology, 54, 557-575.

Rosenberg, M. (1965). Society and the adolescent self-image. Princeton, NJ: Princeton University Press.

Rutter, M. (1986). The developmental psychopathology of depression: Issues and perspectives. En M. Rutter, C.E. Izard y P.B. Read (Eds.), Depression in young people: Developmental and clinical perspectives (pp. 3-32). New York: Guilford Press.

Rutter, M. (1989). Isle of wigth revisited: twenty-five years of child psychiatric epi- 
demiology. Journal of the American Academy of Child Psychiatry, 28, 633-653.

Seisdedos, N. (1990). Adaptación española del cuestionario: State-trait Anxiety Inventory for Children (STAIC). Madrid: TEA.

Seligman, M.E.P. (1975). Helplessness: on depression, development and death. San Francisco, CA: Freeman and Company.

Shaffi, M. y Shaffi, S.L. (1992). Clinical guide to depression in children and adolescents. Washington: American Psychiatric Press.

Spielberger, C.D., Edwards, C.D., Lushene, R.E., Montuori, J. y Platzek, D. (1973). STAIC, State-trait Anxiety Inventory for Children. Consulting Psychologist Press.
Spivack, G. y Shure, M.B. (1974). Social adjustment of young children: $A$ cognitive approach to solving real-life problems. San Francisco: Jossey-Bass.

Tomlinson, C., Bland, L. y Moon, T. (1993). Evaluation utilization; a review of the literarature with implications for gifted education. Journal for the Education of the Gifted, 16, 171-189.

Vostanis, P. y Harrington, R. (1994).Cognitive-behavioral treatment of depressive disorder in child psychiatric patients: Rational and description of treatment package. European Child and Adolescent Psychiatry, 3 , 111-123. 